\title{
Systematic Review of the Relationships between 24-Hour Movement Behaviours and Health Indicators in School-Aged Children from Arab-Speaking Countries
}

\author{
Yazeed A. Alanazi ${ }^{1, *}$, Eduarda Sousa-Sá ${ }^{1,2,3,4}{ }^{\mathbb{D}}$, Kar Hau Chong ${ }^{1}$, Anne-Maree Parrish ${ }^{1} \mathbb{D}$ and \\ Anthony D. Okely 1,5 (D) \\ 1 Early Start and School of Health \& Society, University of Wollongong, Wollongong, NSW 2522, Australia; \\ emdsr885@uowmail.edu.au (E.S.-S.); khc745@uowmail.edu.au (K.H.C.); aparrish@uow.edu.au (A.-M.P.); \\ tokely@uow.edu.au (A.D.O.) \\ 2 Research Centre in Physical Activity, Health and Leisure, Faculty of Sport, University of Porto, \\ 4200-450 Porto, Portugal \\ 3 CIDEFES—Centro de Investigação em Desporto, Educação Física e Exercício e Saúde, Universidade Lusófona, \\ 1749-024 Lisbon, Portugal \\ 4 Laboratory for Integrative and Translational Research in Population Health, 4050-091 Porto, Portugal \\ 5 Illawarra Health and Medical Research Institute, University of Wollongong, \\ Wollongong, NSW 2522, Australia \\ * Correspondence: yana918@uowmail.edu.au
}

\section{check for} updates

Citation: Alanazi, Y.A.; Sousa-Sá, E.; Chong, K.H.; Parrish, A.-M.; Okely, A.D. Systematic Review of the Relationships between 24-Hour Movement Behaviours and Health Indicators in School-Aged Children from Arab-Speaking Countries. Int. J Environ. Res. Public Health 2021, 18 , 8640. https://doi.org/10.3390/ ijerph18168640

Academic Editor: Paul B. Tchounwou

Received: 15 June 2021

Accepted: 7 August 2021

Published: 16 August 2021

Publisher's Note: MDPI stays neutral with regard to jurisdictional claims in published maps and institutional affiliations.

Copyright: (C) 2021 by the authors. Licensee MDPI, Basel, Switzerland. This article is an open access article distributed under the terms and conditions of the Creative Commons Attribution (CC BY) license (https:/ / creativecommons.org/licenses/by/ $4.0 /)$.

\begin{abstract}
The Australian and Canadian 24-hour movement guidelines for children and youth synthesized studies in English and French or other languages (if able to be translated with Google translate) and found very few studies published in English from Arabic countries that examined the relationship between objectively measured sedentary behaviour (SB), sleep and physical activity (PA) and health indicators in children aged 5-12 years. The purpose of this systematic review was to investigate the relationships between 24-hour movement behaviours and health indicators in schoolaged children from Arab-speaking countries. Online databases MEDLINE, EMBASE, SPORTdiscus, CINAHL, PsycINFO and Scopus were searched for English, French and Arabic studies (written in English), while Saudi Digital Library, ArabBase, HumanIndex, KSUP, Pan-Arab Academic Journal, e-Marefa, Al Manhal eLibrary and Google Scholar were searched for Arabic studies. The Grading of Recommendations Assessment, Development and Evaluation framework was used to assess the risk of bias and the quality of evidence for each health indicator. A total of 16 studies, comprising 15,346 participants from nine countries were included. These studies were conducted between 2000 and 2019. In general, low levels of PA and sleep and high SB were unfavourably associated with adiposity outcomes, behavioural problems, depression and low self-esteem. Favourable associations were reported between sleep duration and adiposity outcomes. SB was favourably associated with adiposity outcomes, withdrawn behaviour, attention and externalizing problems. PA was favourably associated with improved self-esteem and adiposity outcomes. Further studies to address the inequality in the literature in the Arab-speaking countries to understand the role of 24-hour movement behaviours and its positive influence on health outcomes across childhood are urgently needed.
\end{abstract}

Keywords: movement behaviours; child; sleep; sedentary behaviour; physical activity; Arab

\section{Introduction}

For years, movement guidelines for children and adolescents have concentrated on moderate- to vigorous-intensity physical activity (MVPA) [1]. However, focusing on PA per se and omitting other movement behaviours, such as SB and sleep, has reduced our perception of how these daily movement behaviours interact to affect children's health [2]. 
Hence, an approach that integrates all components of movement behaviours is required to review their combined influence on health and development [2].

In 2016, the Canadian Society for Exercise Physiology released the world's first integrated 24-hour movement guidelines for children and youth (5-17 years) [3] - a new concept describing the integration of PA, SB and sleep over the 24-hour period. This terminology is regarded as a shift in daily movement behaviour research and illustrates an evolution in PA guidelines [3]. These guidelines were launched based on the results of four systematic reviews, investigating associations between PA, SB, sleep, and movement combinations, each one with health indicators [3]. The authors found that children's total PA was favourably associated with physical, psychological/social, and cognitive health indicators [4]. Higher levels of TV and screen time viewing were associated with unfavourable body composition, cardio-metabolic disease risk scores, hostile behavioural conduct/pro-social behaviour indicators, lower fitness and self-esteem in children [5]. Shorter sleep duration was associated with poorer health outcomes [6]. Children who had higher levels of PA and sleep and less SB had more desirable measures of adiposity and cardiometabolic health when compared with those with a combination of low PA and low sleep and high SB. Similarly, those with high PA and high sleep or high PA and low SB profiles demonstrated favourable health indicators compared with low PA and low sleep, or low PA and high SB profiles [7].

In 2018, the literature on 24-hour movement guidelines was updated as part of the development of Australian 24-Hour Movement Guidelines for Children and Young People [8]. These guidelines were launched as evidence-based guidelines to address movement behaviours observed over the whole day instead of focusing on these behaviours in isolation. Previous studies only captured specific movement behaviours (e.g., MVPA) during waking hours-which accounts for a small portion of children's daily activity $(<5 \%)$ in the 24-hour period; while sleep ( 40\%), SB ( 40\%) and LPA ( 15\%) make up nearly $95 \%$ of the day [2]. Furthermore, these guidelines were launched based on the results of systematic reviews that synthesized studies in English and French or other languages if able to be translated with Google translate, and found very few studies published in English or French from Arabic countries that examined the relationship between objectively measured SB, sleep and PA and health indicators in children aged 5-12 years. The search criteria did not elicit any studies published in Arabic from Arab countries.

Even though more than 140 million children live in the 22 Arabic countries [9], where there are high and increasing levels of childhood obesity and sedentary behaviour [10,11], the lack of evidence from these countries confirms that research is needed to address this gap in the literature, to understand the role of 24-hour movement behaviours and its influence on important health outcomes (obesity, executive functions, motor development and bone health) across the years of primary school. Moreover, it is likely there may be cultural differences in 24-hour movement behaviours in Arabic countries compared to Western countries, necessitating a separate review. Therefore, the purpose of this systematic review was to investigate the relationship between 24-hour movement behaviours and health indicators in school-aged children in Arabic countries.

\section{Methods}

This systematic review was registered with the International Prospective Register of Systematic Reviews (PROSPERO; Registration no. CRD42020143101). It was conducted and reported following the Preferred Reporting Items for Systematic Reviews and MetaAnalyses (PRISMA) statement for reporting systematic reviews and meta-analyses [12]. The protocol of this study was adopted partly from the systematic review performed by Saunders et al. [7].

\subsection{Eligibility Criteria}

Eligible participants included apparently healthy children aged 5 to 12 years old. Overweight and/or obese children were also included. Studies where the sample were aged 
above 12 years or below 5 years were included if the mean age was between 5-12 years. To be included, studies had to be peer-reviewed, published, written in Arabic, English or French and reported subjective or objective measurement of PA or SB or sleep or their combination. Grey literature, student dissertations or conference abstracts were excluded. The main outcomes were adiposity, cardiometabolic biomarkers, fitness, behavioural conduct/pro-social behaviour, emotional regulation/psychological distress, cognition (academic achievement), quality of life and injuries. Secondary outcomes included bone density, motor skill development and self-esteem. The review was limited to full manuscripts. There was no minimum sample size. All study designs were included.

Twenty-four-hour movement behaviours incorporate sleep, SB and PA, which are independently defined as:

Sleep: "a naturally recurring state of body and mind characterized by altered consciousness, relatively inhibited sensory activity, inhibition of nearly all voluntary muscles and reduced interactions with surroundings". [13]

SB: "any waking behaviour characterized by an energy expenditure $\leq 1.5$ metabolic equivalents (METs), while in a sitting, reclining or lying posture". [14]

PA: "any bodily movement produced by skeletal muscles that results in energy expenditure above the resting metabolic rate". [15]

\subsection{Search Strategy}

Six electronic databases were searched from January, 1990 to January, 2021 to identify relevant articles that were written in English or French: MEDLINE, EMBASE, SPORTdiscus, CINAHL, PsycINFO and Scopus. Eight electronic databases were searched for Arabic studies: Saudi Digital Library, ArabBase, Human Index, KSUP, Pan-Arab Academic Journal, e-Marefa, Al Manhal eLibrary and Google Scholar. Search terms can be seen in the Appendix A.

\subsection{Data Extraction}

Studies were imported into Endnote X9 software (Thomson Reuters, San Francisco, CA, USA). After de-duplication, three authors (YA, ESS and KHC) screened titles and abstracts for relevant studies. Full-text copies of the eligible studies were assessed for final inclusion. Any disagreement between the three authors was resolved through a discussion and, when necessary, included a fourth author. The reference lists of all included studies were screened for additional studies not listed in the database search. Data were extracted for each study using an Excel spread sheet; each study included article, author, study design, publication year, location, sample size, age, mean age, gender, outcomes and measures, study instrument and results (Table 1).

\subsection{Quality Assessment}

Three authors (YA, ESS and KHC) independently assessed the risk of bias (ROB) using the GRADE framework (Grading of Recommendations Assessment, Development, and Evaluation), which was also used to assess the quality of evidence for each health indicator. GRADE does not have an official tool for assessing ROB in observational studies but recommends the types of study characteristics to be evaluated [16]. The quality of evidence was assessed for each included study design based on selection bias, attrition bias, detection bias, performance bias, and selective reporting bias. Quality of evidence scores were considered "low" for experimental and observational studies. Scores above $6 / 8$ were considered as having low risk of bias. 
Table 1. Characteristics of included studies.

\begin{tabular}{|c|c|c|c|c|c|c|c|}
\hline $\begin{array}{l}\text { Literature } \\
\text { Reference and Country }\end{array}$ & $\begin{array}{l}\text { Study } \\
\text { Design }\end{array}$ & $\begin{array}{l}\text { Sample Size } \\
\text { (\% Female), } \\
\text { Mean Age or Age } \\
\text { Range (Years) } \\
\end{array}$ & $\begin{array}{l}\text { Type of } \\
\text { Behaviour }\end{array}$ & $\begin{array}{l}\text { Exposure and } \\
\text { Assessment Instrument }\end{array}$ & Outcomes & $\begin{array}{l}\text { Statistical Analysis \& } \\
\text { Confounders (If } \\
\text { Reported) }\end{array}$ & Main Results \\
\hline $\begin{array}{l}\text { AlHazzaa et al., (2019) } \\
\text { Saudi Arabia [17] }\end{array}$ & Cross-sectional & $\begin{array}{l}1033(51.1 \% \text { female }) \\
\text { mean age }=9.2 \pm 1.7\end{array}$ & Sleep & $\begin{array}{l}\text { Sleep: Parent-proxy reported } \\
\text { average sleep duration per night } \\
\text { ( }<9 \text { h vs. } \geq 9 \text { h). }\end{array}$ & $\begin{array}{l}\text { Adiposity: body weight } \\
\text { (kg) and BMI. }\end{array}$ & $\begin{array}{l}\text { Logistic regression } \\
\text { analysis. Confounders: } \\
\text { body weight, age and } \\
\text { gender. }\end{array}$ & $\begin{array}{l}\text { No significant association between } \\
\text { sleep duration and overweight or } \\
\text { obesity status (aOR }=1.00 ; 95 \% \text { CI } \\
0.71 \text { t0 1.64; } p=0.717)\end{array}$ \\
\hline $\begin{array}{l}\text { Al-Hazzaa, (2007) } \\
\text { Saudi Arabia [18] }\end{array}$ & Cross-sectional & $\begin{array}{l}296(100 \% \text { male }) ; \text { mean } \\
\text { age }=10.3 \pm 1.3\end{array}$ & PA & $\begin{array}{l}\text { PA: Pedometer measured steps } \\
\text { taken/day. }\end{array}$ & $\begin{array}{l}\text { Adiposity: BMI, } \\
\text { skinfold measurements } \\
\text { (triceps and } \\
\text { subscapular, body fat \%, } \\
\text { FMI and FFMI. }\end{array}$ & $\begin{array}{l}\text { Pearson's correlation. } \\
\text { Confounders: age, } \\
\text { gender, daily pedometer } \\
\text { counts and total energy } \\
\text { expenditure. }\end{array}$ & $\begin{array}{l}\text { Significant negative associations } \\
\text { between step counts/day and body } \\
\text { fat } \%(\mathrm{r}=-0.207 ; p=0.006), \mathrm{BMI} \\
(\mathrm{r}=-0.198 ; p=0.007), \text { FMI } \\
(\mathrm{r}=-0.214 ; p=0.004) \text {, but not with } \\
\text { FFMI }(\mathrm{r}=-0.089 ; p=0.231) .\end{array}$ \\
\hline $\begin{array}{l}\text { Hassan and Al-Kharusy, } \\
\text { (2000) } \\
\text { Oman [19] }\end{array}$ & Pilot study & $\begin{array}{l}109(100 \% \text { male }) ; \text { mean } \\
\text { age }=9.68 \pm 0.92\end{array}$ & PA and SB & $\begin{array}{l}\text { PA: Leisure time sport activities } \\
\text { personal activity score } \\
\text { (hours/week) assessed with } \\
\text { 1.6-km run/walk. SB: } \\
\text { Parent-proxy reported duration } \\
\text { of TV watching and/or playing } \\
\text { video or computer games. }\end{array}$ & $\begin{array}{l}\text { Fitness: } \\
\text { cardiorespiratory } \\
\text { endurance. Adiposity: } \\
\text { Log sum of } 5 \text { skinfold } \\
\text { measurements (triceps, } \\
\text { subscapular, suprailiac, } \\
\text { abdominal and thigh). }\end{array}$ & $\begin{array}{l}\text { Pearson correlation } \\
\text { coefficients. }\end{array}$ & $\begin{array}{l}\text { Personal activity score has a strong } \\
\text { negative correlation with the time to } \\
\text { complete the } 1.6 \mathrm{~km} \text { run } / \text { walk and } \\
\text { the sum of skinfolds }(r=-0.40 \text {, } \\
-0.42 ; p=0.001) \text {. No significant } \\
\text { associations between TV watching } \\
\text { hours and fitness or fatness }(p=n . \mathrm{r}) \text {. }\end{array}$ \\
\hline $\begin{array}{l}\text { Badawi et al., (2013) } \\
\text { Egypt [21] }\end{array}$ & Cross-sectional & $\begin{array}{l}852(50.2 \% \text { female }) \\
\text { mean age }=9.5 \pm 1.8\end{array}$ & $\mathrm{PA}$ and SB & $\begin{array}{l}\text { PA: Parent-proxy reported } \\
\text { practice of sports, and } \\
\text { transportation to school. SB: } \\
\text { Parent-proxy reported time } \\
\text { spent watching TV. }\end{array}$ & $\begin{array}{l}\text { Adiposity: BMI and } \\
\text { body weight. }\end{array}$ & t-test, ANOVA test. & $\begin{array}{l}\text { Significant association between low } \\
\text { PA and BMI }(p=<0.001) \text {. Significant } \\
\text { association between SB and BMI } \\
(p=<0.001) \text {. }\end{array}$ \\
\hline $\begin{array}{l}\text { Al-Lahham et al., (2019) } \\
\text { Palestine [22] }\end{array}$ & Cross-sectional & $\begin{array}{l}1320(48 \% \text { female }) \\
\text { mean age }=9.5 \pm 1.5\end{array}$ & PA and SB & $\begin{array}{l}\text { PA: Parent-proxy reported daily } \\
\text { PA (min), mode of transport to } \\
\text { school. SB: Parent-proxy } \\
\text { reported screen time (min). }\end{array}$ & $\begin{array}{l}\text { Adiposity: BMI and } \\
\text { body weight. }\end{array}$ & $\begin{array}{l}\text { Chi square test. } \\
\text { Confounders: } \\
\text { transporting means to } \\
\text { school, total screen time, } \\
\text { total PA time and age. }\end{array}$ & $\begin{array}{l}\text { Significant association between } \\
\text { levels of PA (transportation means } \\
\text { only) and BMI ( } p=0.031) \text {. Screen } \\
\text { time had no significant effect on } \\
\text { BMI, however, it had a borderline } \\
\text { effect }(p=0.069) \text {. }\end{array}$ \\
\hline
\end{tabular}


Table 1. Cont.

\begin{tabular}{|c|c|c|c|c|c|c|c|}
\hline $\begin{array}{l}\text { Literature } \\
\text { Reference and Country }\end{array}$ & $\begin{array}{l}\text { Study } \\
\text { Design }\end{array}$ & $\begin{array}{l}\text { Sample Size } \\
\text { (\% Female), } \\
\text { Mean Age or Age } \\
\text { Range (Years) }\end{array}$ & $\begin{array}{l}\text { Type of } \\
\text { Behaviour }\end{array}$ & $\begin{array}{l}\text { Exposure and } \\
\text { Assessment Instrument }\end{array}$ & Outcomes & $\begin{array}{l}\text { Statistical Analysis \& } \\
\text { Confounders (If } \\
\text { Reported) }\end{array}$ & Main Results \\
\hline $\begin{array}{l}\text { Jemaa et al., (2018) } \\
\text { Tunisia [23] }\end{array}$ & Cross-sectional & $\begin{array}{l}40(47.5 \% \text { female }) \text {; mean } \\
\text { age }=9.34 \pm 0.94\end{array}$ & $\mathrm{PA}$ and SB & $\begin{array}{l}\text { PA and SB: Accelerometer } \\
\text { measures (LPA, MPA, VPA, } \\
\text { MVPA); Subjective measures } \\
\text { (mean PA Questionnaire for } \\
\text { Older Children (PAQ-C) score } \\
\text { and intensity classification). }\end{array}$ & Adiposity: \% fat mass. & $\begin{array}{l}\text { Pearson Correlation } \\
\text { coefficient. }\end{array}$ & $\begin{array}{l}\text { The average MVPA showed a } \\
\text { negative significant correlation with } \\
\text { body fat } \%(\mathrm{r}=-0.343, p=0.030) \text {. } \\
\text { The score of PA determined by } \\
\text { PAQ-C was not significantly } \\
\text { correlated with the body fat } \% \\
(\mathrm{r}=-0.227, p=0.158) \text {. }\end{array}$ \\
\hline $\begin{array}{l}\text { Lafta and Kadhim, } \\
\text { (2005) } \\
\text { Iraq [24] }\end{array}$ & Case control & $\begin{array}{l}2084 \text { (male and female); } \\
7-13 \text { (age range) }\end{array}$ & SB & $\begin{array}{l}\text { SB: Parent proxy reported } \\
\text { watching TV ( }>3 \mathrm{~h} / \text { day) via } \\
\text { questionnaire. }\end{array}$ & $\begin{array}{l}\text { Adiposity: BMI-defined } \\
\text { overweight/obese. }\end{array}$ & $\begin{array}{l}\text { Chi-square test. } \\
\text { Confounders: age, birth } \\
\text { rank, type of feeding } \\
\text { during infancy, dietary } \\
\text { pattern, pattern of PA } \\
\text { and working after } \\
\text { school time. }\end{array}$ & $\begin{array}{l}\text { Watching TV ( }>3 \mathrm{~h} / \text { day) was a } \\
\text { significant factor for overweight in } \\
7-9 \text { year males }\left(\chi^{2}=19.69,95 \% \text { CI }\right. \\
1.79 \text { to } 4.97 ; p<0.001) .\end{array}$ \\
\hline $\begin{array}{l}\text { Alam (2008) Saudi } \\
\text { Arabia [25] }\end{array}$ & Cross-sectional & $\begin{array}{l}1072 \text { (100\% female); } \\
8-12 \text { (age range) }\end{array}$ & SB & $\begin{array}{l}\text { SB: Parent proxy reported } \\
\text { duration of TV watching via } \\
\text { questionnaire. }\end{array}$ & $\begin{array}{l}\text { Adiposity: BMI and } \\
\text { body weight. }\end{array}$ & Chi square test. & $\begin{array}{l}\text { Watching TV }(>2 \mathrm{~h} / \text { day }) \text { was } \\
\text { significantly higher among obese } \\
\text { students }\left(\chi^{2}=12.98, p=0.011\right)\end{array}$ \\
\hline
\end{tabular}


Table 1. Cont.

\begin{tabular}{|c|c|c|c|c|c|c|c|}
\hline $\begin{array}{l}\text { Literature } \\
\text { Reference and Country }\end{array}$ & $\begin{array}{l}\text { Study } \\
\text { Design }\end{array}$ & $\begin{array}{l}\text { Sample Size } \\
\text { (\% Female), } \\
\text { Mean Age or Age } \\
\text { Range (Years) }\end{array}$ & $\begin{array}{l}\text { Type of } \\
\text { Behaviour }\end{array}$ & $\begin{array}{l}\text { Exposure and } \\
\text { Assessment Instrument }\end{array}$ & Outcomes & $\begin{array}{l}\text { Statistical Analysis \& } \\
\text { Confounders (If } \\
\text { Reported) }\end{array}$ & Main Results \\
\hline $\begin{array}{l}\text { Al-Kutbe et al., (2017) } \\
\text { Saudi Arabia [27] }\end{array}$ & Cross-sectional & $\begin{array}{l}266(100 \% \text { female); } 8-11 \\
\text { (age range) }\end{array}$ & PA and SB & $\begin{array}{l}\text { PA and SB: Number of steps } \\
\text { taken/day with accelerometer } \\
\text { (WGT3X-BTActigraph). }\end{array}$ & $\begin{array}{l}\text { Adiposity: body weight } \\
(\mathrm{kg}) \text {. }\end{array}$ & $\begin{array}{l}\text { Multiple linear } \\
\text { regression. } \\
\text { Confounders: daily } \\
\text { energy intake, daily } \\
\text { total energy } \\
\text { expenditure, body } \\
\text { weight, age and family } \\
\text { income. }\end{array}$ & $\begin{array}{l}\text { No association between the number } \\
\text { of steps or the time spent in MVPA } \\
\text { and body weight (Beta }=0.034 ; \\
p=0.575,0.368) .\end{array}$ \\
\hline $\begin{array}{l}\text { Al-Hazzaa and } \\
\text { Alrasheedi, (2007) } \\
\text { Saudi Arabia [28] }\end{array}$ & Cross-sectional & $\begin{array}{l}224(51.3 \% \text { female }) \\
\text { mean age }=5.19 \pm 0.85\end{array}$ & $\mathrm{PA}$ and $\mathrm{SB}$ & $\begin{array}{l}\text { PA: Pedometer measured steps } \\
\text { taken/day. SB: Parent proxy } \\
\text { reported duration of TV } \\
\text { watching/day via questionnaire. }\end{array}$ & $\begin{array}{l}\text { Adiposity: BMI, } \\
\text { skinfold measurements } \\
\text { (triceps, subscapular } \\
\text { (sum and ratio), FM \%, } \\
\text { FFM \%, FMI and FFMI. }\end{array}$ & $\begin{array}{l}\text { One-way ANOVA and } \\
\text { post hoc test (Scheffe). } \\
\text { Confounders: body size } \\
\text { for FMI and FFMI only. }\end{array}$ & $\begin{array}{l}\text { No significant differences between } \\
\text { obese and non-obese children in } \\
\text { steps counts/day ( } p=0.109) \text {. No } \\
\text { significant difference between active } \\
\text { and inactive preschool children in } \\
\text { any of the measured anthropometric } \\
\text { and body composition variables } \\
\text { (body weight }(p=0.644) \text {, BMI } \\
(p=0.961) \text {, triceps skinfold } \\
(p=0.975) \text {, subscapular skinfold } \\
(p=0.738) \text {, sum of } 2 \text { skinfolds } \\
(p=0.854) \text {, subscapular/triceps ratio } \\
(p=0.219) \text {, fat } \%(p=0.985) \text {, fat mass } \\
(p=0.664) \text {, fat free mass }(p=0.744) \text {, } \\
\text { FMI }(p=0.850) \text {, FFMI }(p=0.896) \text {. } \\
\text { Obese children spent significantly } \\
\text { more time watching TV } \\
(197.5 \pm 89.3 \text { min/day) than their } \\
\text { non-obese peers } \\
\text { (150.0 } \pm 60.9 \text { min/day) }(p=0.001) .\end{array}$ \\
\hline $\begin{array}{l}\text { Alqaderi et al., (2016) } \\
\text { Kuwait [29] }\end{array}$ & $\begin{array}{l}\text { Longitudinal } \\
\text { study }\end{array}$ & $\begin{array}{l}8317 \text { in } 1 \text { st phase and } \\
6316 \text { in } 2 \text { nd phase } \\
(61.4 \% \text { female); } 8-11 \text { at } \\
\text { visit } 1,10-12 \text { at follow } \\
\text { up (age range) }\end{array}$ & Sleep & $\begin{array}{l}\text { Sleep: Lifestyle habits interview } \\
\text { reported daily sleep hours, TV } \\
\text { and video game use. }\end{array}$ & $\begin{array}{l}\text { Adiposity: Waist } \\
\text { circumference. }\end{array}$ & $\begin{array}{l}\text { Multilevel longitudinal } \\
\text { linear regression model. } \\
\text { Confounders: age and } \\
\text { gender. }\end{array}$ & $\begin{array}{l}\text { Short sleep duration was } \\
\text { significantly associated with } \\
\text { increased waist circumference } \\
\text { (beta =-0.11; } 95 \% \text { CI } 0.14 \text { to } 0.17 \text {; } \\
p=<0.05 \text { ). }\end{array}$ \\
\hline
\end{tabular}


Table 1. Cont.

\begin{tabular}{|c|c|c|c|c|c|c|c|}
\hline $\begin{array}{l}\text { Literature } \\
\text { Reference and Country }\end{array}$ & $\begin{array}{l}\text { Study } \\
\text { Design }\end{array}$ & $\begin{array}{l}\text { Sample Size } \\
\text { (\% Female), } \\
\text { Mean Age or Age } \\
\text { Range (Years) } \\
\end{array}$ & $\begin{array}{l}\text { Type of } \\
\text { Behaviour }\end{array}$ & $\begin{array}{l}\text { Exposure and } \\
\text { Assessment Instrument }\end{array}$ & Outcomes & $\begin{array}{l}\text { Statistical Analysis \& } \\
\text { Confounders (If } \\
\text { Reported) }\end{array}$ & Main Results \\
\hline $\begin{array}{l}\text { Al-Ghamdi, (2013) } \\
\text { Saudi Arabia [30] }\end{array}$ & Case control & $\begin{array}{l}397(49.3 \% \text { female); } \\
\text { mean age = } 11.4 \text { (SD: } \\
n . r .)\end{array}$ & $\mathrm{PA}$ and $\mathrm{SB}$ & $\begin{array}{l}\text { PA and SB: questionnaire } \\
\text { (interview) reported watching } \\
\text { TV ( }>3 \mathrm{~h} \text { /day) and daily } \\
\text { exercise. }\end{array}$ & Adiposity: BMI. & $\begin{array}{l}\text { Chi-square test. } \\
\text { Confounders: TV, VG } \\
\text { time/day, age, daily } \\
\text { exercise/day. }\end{array}$ & $\begin{array}{l}\text { Watching TV }(>3 \mathrm{~h} / \text { day), especially } \\
\text { over the weekend, was significantly } \\
\text { associated with childhood obesity } \\
\left(\chi^{2}=4.136, p=0.042\right) \text {. No significant } \\
\text { associations between the rate of } \\
\text { exercising at school, home and } \\
\text { outdoors and obesity }\left(\chi^{2}=1.248,\right. \\
1.032,2.604 ; p=0.870,0.905,0.626) .\end{array}$ \\
\hline $\begin{array}{l}\text { Yousef et al., (2013) } \\
\text { UAE [31] }\end{array}$ & Cross-sectional & $\begin{array}{l}197(34 \% \text { female }) ; \text { mean } \\
\text { age }=8.7 \pm 2.1\end{array}$ & SB & $\begin{array}{l}\text { SB: Parent proxy reported } \\
\text { watching TV ( }>2 \mathrm{~h} / \text { day). }\end{array}$ & Behavioral problems. & $\begin{array}{l}\text { Chi square test, logistic } \\
\text { regression. } \\
\text { Confounders: birth } \\
\text { order and number of } \\
\text { siblings. }\end{array}$ & $\begin{array}{l}\text { Watching TV/video game }>2 \mathrm{~h} / \text { day } \\
\text { was associated with withdrawn } \\
\text { behavior }(\mathrm{OR}=0.275 ; 95 \% \text { CI } 0.106 \\
\text { to } 0.712 ; p=0.008), \text { attention } \\
\text { problem }(\mathrm{OR}=0.480 ; 95 \% \text { CI } 0.241 \text { to } \\
0.956 ; p=0.037), \text { externalizing } \\
\text { problems }(\mathrm{OR}=0.393 ; 95 \% \text { CI } 0.201 \\
\text { to } 0.771 ; p=0.007) \text { and Child } \\
\text { Behavior Checklist total score } \\
(\mathrm{OR}=0.441 ; 95 \% \text { CI } 0.229 \text { to } 0.848 ; \\
p=0.014) .\end{array}$ \\
\hline $\begin{array}{l}\text { Zayed and Kilani, (2014) } \\
\text { Oman [32] }\end{array}$ & Cross-sectional & $\begin{array}{l}165 \text { (100\% female); } \\
10-13 \text { (age range) }\end{array}$ & PA & $\begin{array}{l}\text { PA: number of occurrences and } \\
\text { the duration of the practice of } \\
\text { PA per week assessed with PA } \\
\text { interview questionnaire. }\end{array}$ & $\begin{array}{l}\text { Depression and low } \\
\text { self-esteem. }\end{array}$ & $\begin{array}{l}\text { One-way ANOVA and } \\
\text { post hoc test (Scheffe). }\end{array}$ & $\begin{array}{l}\text { Regular PA was significantly } \\
\text { associated with improved } \\
\text { self-esteem; differences were seen } \\
\text { between those who never exercised } \\
\text { and those who exercised regularly } \\
\text { (mean square = 358.257; F = 4.787; } \\
p=0.10 \text { ). }\end{array}$ \\
\hline
\end{tabular}

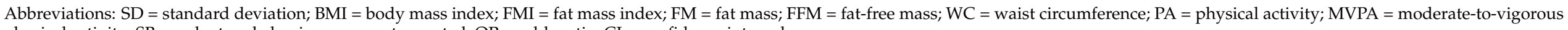
physical activity; $\mathrm{SB}=$ sedentary behavior; n.r. = not reported; $\mathrm{OR}=$ odds ratio; $\mathrm{CI}=$ confidence interval. 


\section{Results}

A total of 612 studies were eligible for inclusion. After title and abstract screening, 102 studies were assessed for full-text review. Of those, 86 were excluded for the following reasons: did not contain measures of PA or SB or sleep as an independent variable $(n=21)$; did not contain a measure of a health indicator and its association with PA or SB or sleep $(n=13)$; out of range for age $(n=37)$; dissertation $(n=7)$; studies conducted in non-Arab countries $(n=4)$; unavailability of the full article $(n=3)$; and lack of statistical data $(n=1)$. After all exclusions, 16 studies met the inclusion criteria (Figure 1). These studies provided results from 15,346 participants from 9 Arabic countries: Saudi Arabia, $n=6$, United Arab Emirates, $n=1$, Egypt, $n=2$, Oman, $n=2$, Kuwait, $n=1$, Iraq, $n=1$, Tunisia, $n=1$, Qatar, $n=1$ and Palestine, $n=1$ (Table 1). Of all included studies, 12 were cross-sectional, two were case-control, one was longitudinal and one was a pilot study. These studies were conducted between 2000 and 2019 and included children between 3.4 and 14 years of age (mean age 5.19-11.4 years). Sample sizes ranged from 40 to 8317 participants. Out of the 16 included studies, 14 reported data on adiposity [17-30], one on behavioural problems [31], one on depression and low self-esteem [32] and one on fitness [19]. Out of the sixteen studies included in this review, eight studies $(50 \%)$ were classified as having a low ROB and eight as having a high ROB (50\%). All studies had a reliable and/or valid tool to assess movement behaviours and health outcomes. The criteria used to assess ROB can be seen in Table 2. It was not possible to conduct meta-analyses due to heterogeneity of the data, therefore, narrative syntheses were conducted.

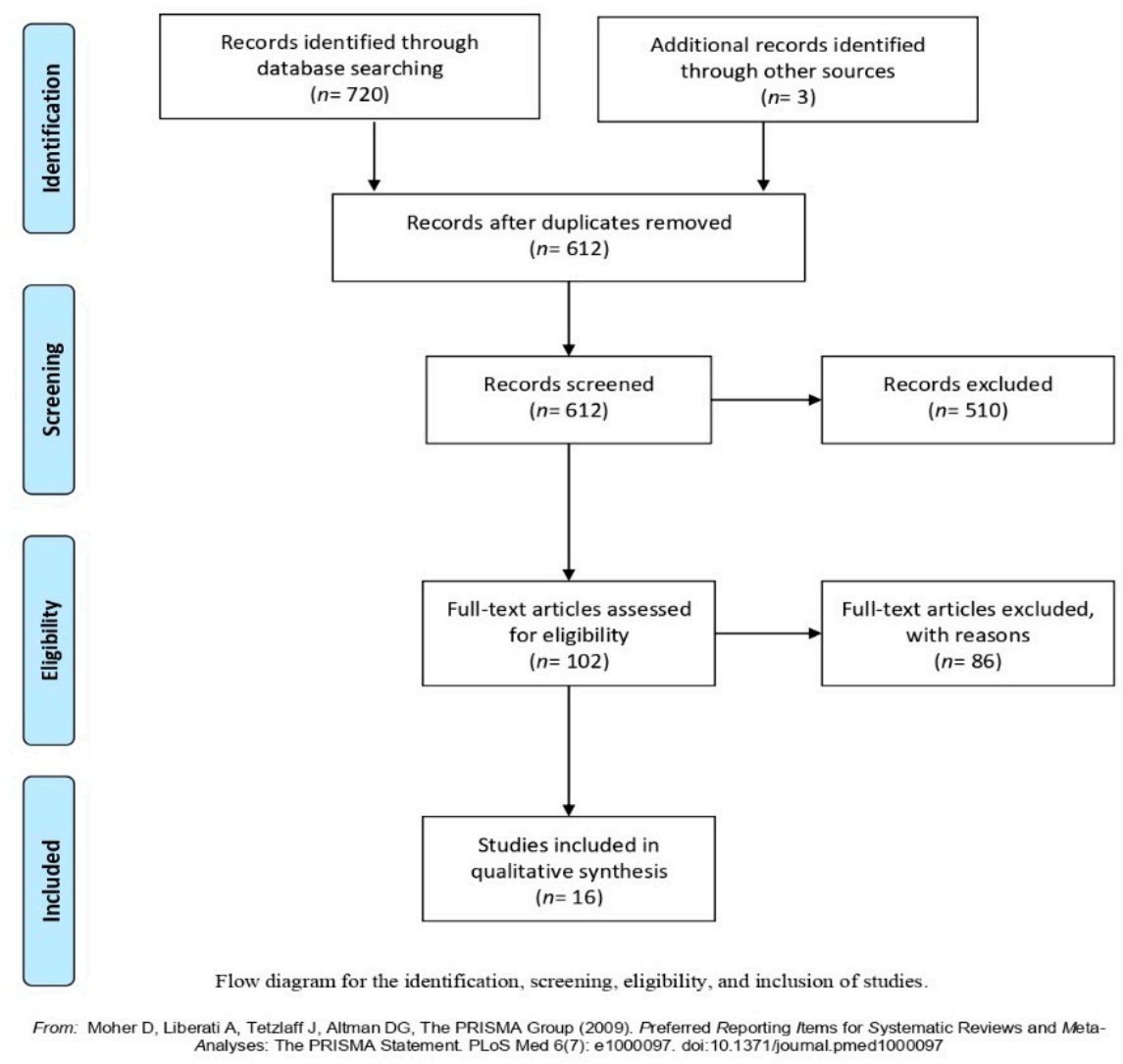

Figure 1. PRISMA Flow Diagram. 
Table 2. Risk of bias for included studies.

\begin{tabular}{|c|c|c|c|c|c|c|c|c|c|c|}
\hline \multirow[b]{2}{*}{ Study } & \multicolumn{2}{|c|}{ 1. Selection Bias } & \multirow{2}{*}{$\begin{array}{c}\text { 2. Attrition Bias } \\
\text { Did an Adequate } \\
\text { Proportion (At Least } 70 \% \text { ) } \\
\text { of Those Consenting to } \\
\text { Participate in the Study } \\
\text { Have Complete Data? } \\
\text { (Incomplete Follow-Up; } \\
\text { High Loss to Follow-Up; } \\
\text { Missing Data) }\end{array}$} & \multicolumn{2}{|c|}{ 3. Detection Bias } & \multicolumn{2}{|c|}{ 4. Performance Bias } & \multirow{2}{*}{$\begin{array}{c}\text { 5. Selective } \\
\text { Reporting Bias } \\
\text { Did the Study } \\
\text { Have Complete } \\
\text { Data and/or } \\
\text { Reports All } \\
\text { Outcomes and } \\
\text { Not Others } \\
\text { Based on the } \\
\text { Results? }\end{array}$} & \multirow[b]{2}{*}{ Score * } & \multirow[b]{2}{*}{$\begin{array}{l}\text { ROB } \\
\text { Qualit }\end{array}$} \\
\hline & $\begin{array}{l}\text { Clear Criteria } \\
\text { for Those } \\
\text { included and/or } \\
\text { Excluded? }\end{array}$ & $\begin{array}{l}\text { Was the } \\
\text { Sample } \\
\text { Randomly } \\
\text { Selected? }\end{array}$ & & $\begin{array}{l}\text { Did the Study } \\
\text { Report the } \\
\text { Sources and } \\
\text { Details of the } \\
\text { Tool Used in } \\
\text { the Study to } \\
\text { Assess The } \\
\text { Outcomes? }\end{array}$ & $\begin{array}{l}\text { Was the Tool } \\
\text { Used in the } \\
\text { Study to Assess } \\
\text { the Outcomes } \\
\text { Reliable and/or } \\
\text { Valid? }\end{array}$ & $\begin{array}{l}\text { Did the Study } \\
\text { Report the Sources } \\
\text { and Details of the } \\
\text { Measurement Tool } \\
\text { Used in the Study } \\
\text { for Movement } \\
\text { Behaviours? }\end{array}$ & $\begin{array}{c}\text { Were the } \\
\text { Measurements } \\
\text { of Movement } \\
\text { Behaviours in } \\
\text { This Study } \\
\text { Reliable and/or } \\
\text { Valid? }\end{array}$ & & & \\
\hline $\begin{array}{c}\text { Al-Hazzaa et al., } \\
2019 \text { [17] }\end{array}$ & 1 & 1 & 1 & 1 & 0 & 1 & 0 & 1 & $6 / 8$ & Low \\
\hline Al-Hazzaa, 2007 [18] & 0 & 0 & 0 & 1 & 1 & 1 & 1 & 1 & $5 / 8$ & High \\
\hline $\begin{array}{c}\text { Hassan and } \\
\text { Al-Kharusy, } 2000 \\
{[19]}\end{array}$ & 0 & 0 & 1 & 1 & 1 & 0 & 0 & 1 & $4 / 8$ & High \\
\hline $\begin{array}{l}\text { Hadhood et al., } 2016 \\
\text { [20] }\end{array}$ & 1 & 1 & 1 & 1 & 1 & 0 & 0 & 1 & $6 / 8$ & Low \\
\hline $\begin{array}{l}\text { Al-Lahham et al., } \\
2019 \text { [22] }\end{array}$ & 1 & 0 & 0 & 1 & 1 & 0 & 0 & 1 & $4 / 8$ & High \\
\hline $\begin{array}{c}\text { Jemaa et al., } 2018 \\
\text { [23] }\end{array}$ & 1 & 0 & 1 & 1 & 1 & 1 & 1 & 0 & $6 / 8$ & Low \\
\hline $\begin{array}{c}\text { Lafta and Kadhim, } \\
2005[24]\end{array}$ & 1 & 1 & 1 & 1 & 0 & 0 & 0 & 1 & $5 / 8$ & High \\
\hline Alam, 2008 [25] & 1 & 0 & 1 & 0 & 1 & 0 & 0 & 1 & $4 / 8$ & High \\
\hline $\begin{array}{c}\text { Arora et al., } 2018 \\
\text { [26] }\end{array}$ & 1 & 0 & 1 & 1 & 1 & 1 & 1 & 1 & $7 / 8$ & Low \\
\hline $\begin{array}{c}\text { Al-Kutbe et al., } 2017 \\
\text { [27] }\end{array}$ & 1 & 0 & 0 & 1 & 1 & 1 & 1 & 1 & $6 / 8$ & Low \\
\hline
\end{tabular}


Table 2. Cont.

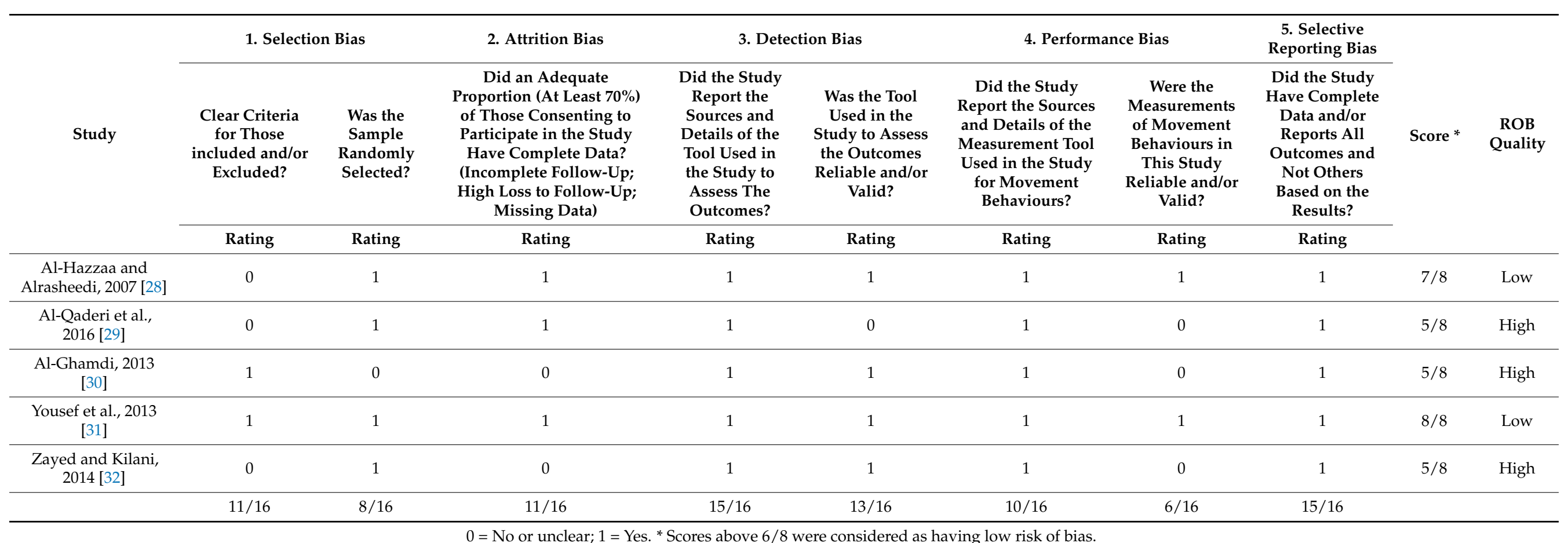

$0=$ No or unclear; $1=$ Yes. ${ }^{*}$ Scores above $6 / 8$ were considered as having low risk of bias. 


\subsection{Measurement of Movement Behaviours}

Sleep was objectively measured by wrist actigraphy in one study [26] and subjectively measured using questionnaires in two studies $[17,29]$. SB was measured using screen time in eight studies $[19,21,22,24,25,28,30,31]$ and objectively measured using accelerometers in two studies $[23,27]$, and by both methods (wrist actigraphy and a questionnaire) in one study [26].

PA was measured using an accelerometer in one study [27] and by pedometer in two studies $[18,28]$; whereas five studies measured it subjectively using parent proxyreports [20-22]. Two studies combined both report and device-based methods: accelerometers plus questionnaire [23] and cardiorespiratory endurance (1.6 km run/walk) plus questionnaire [19].

\subsection{Health Indicators}

\subsubsection{Adiposity}

As shown in Table 3, adiposity was assessed through the following indicators: BMI, body weight, $\%$ fat mass, BMI z-score, and waist circumference. It was reported in 14 studies, of which 10 were cross-sectional [17,18,20-23,25-28], one was longitudinal [29], two were case control $[24,30]$ and one was a pilot study [19]. Three studies investigated the relationship between sleep and adiposity outcomes. Of the three studies, two reported significant positive associations [26,29] while one found no significant relationship [17].

Nine studies examined the relationship between SB and adiposity outcomes. SB was positively associated with adiposity outcomes in six of the nine studies [21,24-26,28,30]. The remaining three studies found no associations with adiposity outcomes $[22,23,27]$. Nine studies examined the adiposity relationship with PA. Of the nine studies, five found favourable associations between adiposity outcomes and PA $[18,19,21-23]$ while four studies reported null associations $[20,27,28,30]$.

\subsubsection{Behavioural Problems}

Behavioural problems were reported in only one cross-sectional study [31] involving 197 subjects (mean age $8.7 \pm 2.1$ ), which studied the relationship between SB and behavioural problems in school-aged children. The results showed that watching TV/playing VG for more than two hours were positively associated with withdrawn behaviour, attention and externalizing problems.

\subsubsection{Depression and Low Self-Esteem}

One study examined the association between PA and depression and low selfesteem [32]; it involved 165 female subjects with age range of 10-13 years. The results indicated that regular PA (number of occurrences and the duration of the practice of PA per week) was significantly associated with improved self-esteem.

\subsubsection{Fitness}

One study assessed the relationship between SB and fitness and reported null associations [19]. The results showed that the personal activity score had a strong negative correlation with the time to complete the $1.6 \mathrm{~km}$ run/walk and the sum of skinfolds. There were no significant associations between TV watching hours and fitness or fatness.

There were no studies investigating the associations with the rest of the primary outcomes, namely cardio metabolic biomarkers, psychological distress, cognition (academic achievement), quality of life, injuries, nor on secondary outcomes including bone density and motor skill development. 
Table 3. Results of studies.

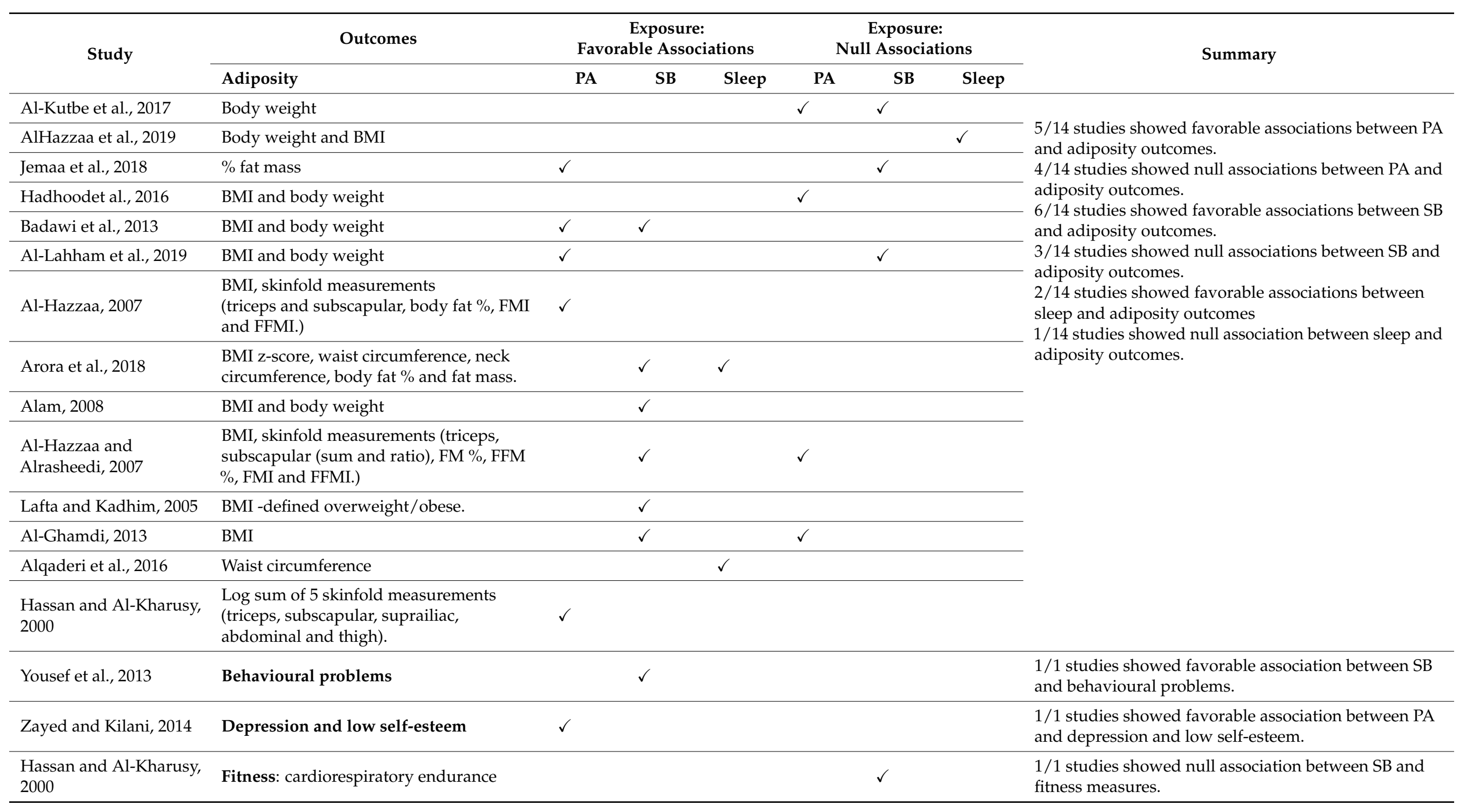




\section{Discussion}

This study systematically reviewed the relationships between the movement behaviours of physical activity, sedentary behaviours and sleep and health indicators among school-aged children in Arab-speaking countries. Most of the included studies in this review were cross-sectional (75\%). The sample sizes ranged from 40 to 8317 participants. These studies reported mostly favourable and some null associations between PA, SB, and sleep and adiposity, behavioural problems, depression and low self-esteem and fitness outcomes. Low levels of PA and sleep and high SB were associated with higher levels of adiposity, behavioural problems, depression and low self-esteem.

Reasons for the small number of studies investigating these movement behaviours in school-aged children in Arab-speaking countries are that this field of research is still in its early stages of development in these countries, with most child health research focusing on more pressing issues such as infectious diseases. In addition, the unstable political environment in some of the Arab countries, has made conducting such research challenging [33]. The availability of funds is another reason that limited the number of these types of studies. For instance, in 2013, the gross domestic expenditure on research and development (GERD) in North America was $\$ 427$ billion (28.9\%) of the worldwide GERD ( $\$ 1477.7$ billion), while Arab countries collectively only spent $\$ 15.4$ billion (1\%) [34]. Finally, although the Arabic databases were searched for relevant studies in this systematic review, there might be some studies that could not be identified due to the small number of Arabic databases available.

Existing Arabic studies assessed movement behaviours in isolation from each other. Of these studies, most used subjective methods to assess PA, sleep and SB. On the other hand, the Canadian [3,4,6,7] and the Australian 24-Hour Movement Guidelines [8] indicated that focusing on movement behaviours across the entire day is more important than focusing on movement behaviours in isolation. For example, a Canadian study investigating the health outcomes associated with meeting the 24-hour movement behaviour guidelines for children and youth showed that meeting none, one and two recommendations were associated with higher BMI z-score, waist circumference, behavioural strengths and difficulties scores and lower aerobic fitness in a gradient pattern $\left(p_{\text {trend }}<0.05\right)$, while meeting all the guidelines during a 24-hour period was associated with better health [35].

Furthermore, due to the prior emphasis on MVPA [1] and the common use of subjective assessments of PA [4], no study in the present review examined different PA intensities such as light-intensity physical activity (LPA), although emerging evidence suggests that LPA may provide some important health benefits for children and adolescents [2-4]. Moreover, children cannot participate in MVPA during all waking hours. Therefore, engaging in LPA (e.g., walking) is considered achievable and an easier way to reduce SB, that also provides health benefits [2].

Few studies in this systematic review assessed the relationship between sleep and adiposity outcomes. A possible explanation of this gap is that sleep and SB are new areas of research in this region when compared to the PA field as the SB included studies were published between 2005-2019, while sleep studies were published in the last five years. Therefore, the Arab countries are urgently in need to conduct more studies that focus on sleep and SB to better understand their impact on school-aged children's health.

Despite the importance of the weather and its impact on movement behaviours [36], no study in the present review assessed the relationship between 24-hour movement behaviours and climatic factors, although most of the Arab countries have a hot and dry climate [37]. Previous research in other countries indicates that children's PA levels are affected by seasonal periods across the year and this varies between countries, with PA levels decreasing in specific climatic conditions such as winter, summer, sandstorms areas, humidity and rain [38,39]. Moreover, extreme weather conditions (high or low temperatures) increase SB [40] and decrease sleep efficiency [41], therefore, conducting more studies investigating the association between the weather and movement behaviours 
and potential interventions may help children in these regions to meet movement behaviour recommendations.

\subsection{Areas for Future Research}

The lack of evidence from the Arab countries confirms that research is needed to address the inequality in the literature, especially with the high and still increasing levels of childhood obesity and SB in the 22 countries. Moreover, it is important to use different types of study designs (longitudinal and experimental) with larger sample sizes to better understand the role of 24-hour movement behaviours, to improve health outcomes. Studies included in this review focused on obesity, however, the field of PA is broader than this health outcome, therefore, it is recommended to conduct more studies reflecting on all movement behaviours across the 24-hour period.

\subsection{Strengths and Limitations}

To our knowledge, the present review is the first study investigating current research assessing the association between movement behaviours and health indicators in schoolaged children in the Arab-speaking countries. A lack of Arabic databases is also a potential limitation. Meta-analyses were not possible to conduct due to heterogeneity of the data, therefore, narrative syntheses were conducted.

\section{Conclusions}

Most of the included studies reported favourable associations between movement behaviours and health outcomes. Low levels of PA and sleep and high SB were unfavourably associated with adiposity outcomes, behavioural problems, depression and low self-esteem. Further studies to address the inequality in the literature in the Arab-speaking countries to understand the role of 24-hour movement behaviours and its positive influence on health outcomes across the early years of primary school are urgently needed. Based on the differences between societies and their needs, as well as environmental differences, it might be beneficial to also understand associations between weather conditions and children's movement behaviours. Conducting more studies on different types/intensities of PA, SB and sleep for both boys and girls, and using different types of study designs (longitudinal and experimental) with larger sample sizes will improve the quality of future studies.

Author Contributions: Conceptualization, Y.A.A., E.S.-S., K.H.C., A.-M.P. and A.D.O.; methodology, Y.A.A., E.S.-S., K.H.C., A.-M.P. and A.D.O.; writing-original draft preparation, Y.A.A.; writingreview and editing, all authors. All authors have read and agreed to the published version of the manuscript.

Funding: This research received no external funding.

Institutional Review Board Statement: The study was conducted according to the guidelines of the Declaration of Helsinki. PROSPERO registration number: CRD42020143101.

Informed Consent Statement: Not applicable.

Acknowledgments: Y.A.A. is supported by the Saudi Arabian Cultural Mission, Canberra, Australia. A.D.O. is supported by a National Health and Medical Research Council of Australia Investigator Grant.

Conflicts of Interest: The authors declare that they have no competing interests.

\section{Appendix A. Supplementary Information}

"Physical activit" OR "Movement behavi*" OR "physical inactivity" OR exercise OR "physical fitness" OR "energy expenditure" OR "sedentary" OR sit* OR sitting OR lifestyle OR "television view" ${ }^{*}$ " OR "tv view*" OR "screen time" OR " electronic media" OR Sleep* OR Bedtime OR "Bed time" OR Nap* OR "Time on bed" OR "Night rest" Child* Arab* OR " Saudi Arabia" OR "United Arab Emirates" OR Bahrain OR Kuwait OR Oman OR Qatar OR Egypt OR Sudan OR Palestine OR Jordan OR Iraq OR Lebanon OR Syria 
OR Yemen OR Libya OR Morocco OR Tunisia OR Algeria OR Comoros OR Djibouti OR Mauritania OR Somalia.

\section{References}

1. Tremblay, M.S.; Colley, R.C.; Saunders, T.; Healy, G.; Owen, N. Physiological and health implications of a sedentary lifestyle. Appl. Physiol. Nutr. Metab. 2010, 35, 725-740. [CrossRef] [PubMed]

2. Chaput, J.-P.; Carson, V.; Gray, C.E.; Tremblay, M.S. Importance of All Movement Behaviors in a 24 Hour Period for Overall Health. Int. J. Environ. Res. Public Health 2014, 11, 12575-12581. [CrossRef] [PubMed]

3. Tremblay, M.S.; Carson, V.; Chaput, J.-P.; Gorber, S.C.; Dinh, T.; Duggan, M.; Faulkner, G.; Gray, C.E.; Gruber, R.; Janson, K.; et al. Canadian 24-Hour Movement Guidelines for Children and Youth: An Integration of Physical Activity, Sedentary Behaviour, and Sleep. Appl. Physiol. Nutr. Metab. 2016, 41, S311-S327. [CrossRef]

4. Poitras, V.J.; Gray, C.; Borghese, M.M.; Carson, V.; Chaput, J.-P.; Janssen, I.; Katzmarzyk, P.; Pate, R.R.; Gorber, S.C.; Kho, M.; et al. Systematic review of the relationships between objectively measured physical activity and health indicators in school-aged children and youth. Appl. Physiol. Nutr. Metab. 2016, 41, S197-S239. [CrossRef]

5. Carson, V.; Hunter, S.; Kuzik, N.; Gray, C.; Poitras, V.J.; Chaput, J.-P.; Saunders, T.J.; Katzmarzyk, P.; Okely, A.; Gorber, S.C.; et al. Systematic review of sedentary behaviour and health indicators in school-aged children and youth: An update. Appl. Physiol. Nutr. Metab. 2016, 41, S240-S265. [CrossRef] [PubMed]

6. Chaput, J.-P.; Gray, C.; Poitras, V.J.; Carson, V.; Gruber, R.; Olds, T.; Weiss, S.K.; Gorber, S.C.; Kho, M.; Sampson, M.; et al. Systematic review of the relationships between sleep duration and health indicators in school-aged children and youth. Appl. Physiol. Nutr. Metab. 2016, 41, S266-S282. [CrossRef] [PubMed]

7. Saunders, T.J.; Gray, C.; Poitras, V.J.; Chaput, J.-P.; Janssen, I.; Katzmarzyk, P.; Olds, T.; Gorber, S.C.; Kho, M.; Sampson, M.; et al. Combinations of physical activity, sedentary behaviour and sleep: Relationships with health indicators in school-aged children and youth. Appl. Physiol. Nutr. Metab. 2016, 41, S283-S293. [CrossRef] [PubMed]

8. Okely, A.; Ghersi, D.; Loughran, S.; Cliff, D.; Shilton, T.; Jones, R. Australian 24-hour movement guidelines for children (5-12 years) and young people (13-17 years): An integration of physical activity, sedentary behaviour. Canberra: Aust. Gov. 2019. [CrossRef]

9. Child labour in Arab States (IPEC). International Programme on the Elimination of Child Labour (IPEC). Available online: https://www.ilo.org/ipec/Regionsandcountries/arab-states/lang--en/index.htm (accessed on 8 January 2020).

10. Sharara, E.; Akik, C.; Ghattas, H.; Obermeyer, C.M. Physical inactivity, gender and culture in Arab countries: A systematic assessment of the literature. BMC Public Health 2018, 18, 639. [CrossRef]

11. Farrag, N.S.; Cheskin, L.J.; Farag, M.K. A systematic review of childhood obesity in the Middle East and North Africa (MENA) region: Prevalence and risk factors meta-analysis. Adv. Pediatr. Res. 2017, 4, 8. [CrossRef]

12. Liberati, A.; Altman, D.G.; Tetzlaff, J.; Mulrow, C.; Gøtzsche, P.C.; Ioannidis, J.P.A.; Clarke, M.; Devereaux, P.J.; Kleijnen, J.; Moher, D. The PRISMA statement for reporting systematic reviews and meta-analyses of studies that evaluate health care interventions: Explanation and elaboration. J. Clin. Epidemiol. 2009, 62, e1-e34. [CrossRef]

13. Chaput, J.; Saunders, T.; Carson, V. Interactions between sleep, movement and other non-movement behaviours in the pathogenesis of childhood obesity. Obes. Rev. 2017, 18,7-14. [CrossRef]

14. Tremblay, M.S.; Aubert, S.; Barnes, J.D.; Saunders, T.J.; Carson, V.; Latimer-Cheung, A.E.; Chastin, S.F.M.; Altenburg, T.M.; Chinapaw, M.J.M.; On Behalf of Sbrn Terminology Consensus Project Participants. Sedentary Behavior Research Network (SBRN)-Terminology Consensus Project process and outcome. Int. J. Behav. Nutr. Phys. Act. 2017, 14, 75. [CrossRef]

15. Caspersen, C.J.; Powell, K.E.; Christenson, G.M. Physical activity, exercise, and physical fitness: Definitions and distinctions for health-related research. Public Health Rep. 1985, 100, 126-131.

16. Guyatt, G.; Oxman, A.D.; Akl, E.A.; Kunz, R.; Vist, G.; Brozek, J.; Norris, S.; Falck-Ytter, Y.; Glasziou, P.; Debeer, H. GRADE guidelines: 1. Introduction-GRADE evidence profiles and summary of findings tables. J. Clin. Epidemiology 2011, 64, 383-394. [CrossRef]

17. Al-Hazzaa, H.M.; Alhussain, M.; Alhowikan, A.M.; Obeid, O.A. Insufficient Sleep Duration and Its Association with Breakfast Intake, Overweight/Obesity, Socio-Demographics and Selected Lifestyle Behaviors among Saudi School Children. Nat. Sci. Sleep 2019, 11, 253-263. [CrossRef]

18. Al-Hazzaa, H.M. Pedometer-determined physical activity among obese and non-obese 8- to 12-year-old Saudi schoolboys. J. Physiol. Anthr. 2007, 26, 459-465. [CrossRef]

19. Hassan, M.O.; Al-Kharusy, W. Physical fitness and fatness among Omani schoolboys: A pilot study. J. Sci. Res. Med Sci. 2000, 2, 37-41.

20. Hadhood, S.E.S.A.; Ali, R.A.E.; Mohamed, M.M.; Mohammed, E.S. Prevalence and Correlates of Overweight and Obesity among School Children in Sohag, Egypt. Open J. Gastroenterol. 2017, 7, 75-88. [CrossRef]

21. Badawi, N.E.-S.; Barakat, A.A.; El Sherbini, S.A.; Fawzy, H.M. Prevalence of overweight and obesity in primary school children in Port Said city. Egypt. Pediatr. Assoc. Gaz. 2013, 61, 31-36. [CrossRef]

22. Al-Lahham, S.; Jaradat, N.; Altamimi, M.; Anabtawi, O.; Irshid, A.; Alqub, M.; Dwikat, M.; Nafaa, F.; Badran, L.; Mohareb, R.; et al. Prevalence of underweight, overweight and obesity among Palestinian school-age children and the associated risk factors: A cross sectional study. BMC Pediatr. 2019, 19, 1-13. [CrossRef] 
23. Ben Jemaa, H.; Mankaï, A.; Mahjoub, F.; Kortobi, B.; Khlifi, S.; Draoui, J.; Minaoui, R.; Karmous, I.; Ben Hmad, H.; Ben Slama, F.; et al. Physical Activity Level Assessed by Accelerometer and PAQ-C in Tunisian Children. Ann. Nutr. Metab. 2018, 73, 234-240. [CrossRef]

24. Lafta, R.K.; Kadhim, M.J. Childhood obesity in Iraq: Prevalence and possible risk factors. Ann. Saudi Med. 2005, 25, 389-393. [CrossRef]

25. Alam, A.A. Obesity among female school children in North West Riyadh in relation to affluent lifestyle. Saudi Med. J. 2008, 29, 1139-1144.

26. Arora, T.; Gad, H.; Omar, O.M.; Choudhury, S.; Chagoury, O.; Sheikh, J.; Taheri, S. The associations among objectively estimated sleep and obesity indicators in elementary schoolchildren. Sleep Med. 2018, 47, 25-31. [CrossRef]

27. Al-Kutbe, R.; Payne, A.; De Looy, A.; Rees, G.A. A comparison of nutritional intake and daily physical activity of girls aged 8-11 years old in Makkah, Saudi Arabia according to weight status. BMC Public Health 2017, 17, 592. [CrossRef]

28. Al-Hazzaa, H.M.; A Al-Rasheedi, A. Adiposity and physical activity levels among preschool children in Jeddah, Saudi Arabia. Saudi Med. J. 2007, 28, 766.

29. Alqaderi, H.; Goodson, J.M.; Subramanian, S.V.; Tavares, M. Short Sleep Duration and Screen-Based Activities: A Longitudinal Multilevel Analysis. Am. J. Lifestyle Med. 2016, 12, 340-348. [CrossRef] [PubMed]

30. Al-Ghamdi, S.H. The association between watching television and obesity in children of school-age in Saudi Arabia. J. Fam. Community Med. 2013, 20, 83-89. [CrossRef] [PubMed]

31. Yousef, S.; Eapen, V.; Zoubeidi, T.; Mabrouk, A. Behavioral correlation with television watching and videogame playing among children in the United Arab Emirates. Int. J. Psychiatry Clin. Pr. 2014, 18, 203-207. [CrossRef] [PubMed]

32. Zayed, K.; Kilani, H. Physical activity is associated with depression and self-esteem levels among Omani female children. Can. J. Clin. Nutr. 2014, 2, 15-28.

33. COVID-19 on the Arab Region An Opportunity to Build Back Better. 2020. Available online: https://unsdg.un.org/resources/ policy-brief-impact-covid-19-arab-region-opportunity-build-back-better (accessed on 10 January 2021).

34. Baskaran, A. UNESCO Science Report: Towards 2030. Inst. Econ. 2016, 8, 125-127.

35. Carson, V.; Chaput, J.-P.; Janssen, I.; Tremblay, M.S. Health associations with meeting new 24-hour movement guidelines for Canadian children and youth. Prev. Med. 2017, 95, 7-13. [CrossRef]

36. Turrisi, T.B.; Bittel, K.M.; West, A.B.; Hojjatinia, S.; Hojjatinia, S.; Mama, S.K.; Lagoa, C.M.; Conroy, D.E. Seasons, weather, and device-measured movement behaviors: A scoping review from 2006 to 2020. Int. J. Behav. Nutr. Phys. Act. 2021, 18, 1-26. [CrossRef]

37. Elasha, B.O. Mapping of Climate Change Threats and Human Development Impacts in the Arab Region; UNDP Arab Development Report-Research Paper Series; UNDP Regiona Bureau for the Arab States: Amman, Jordan, 2010. Available online: https:/ / www.arabstates.undp.org/content/rbas/en/home/library/huma_development/mapping-of-climate-changethreats-and-human-development-impacts-.html (accessed on 6 January 2021).

38. Al-Mohannadi, A.; Moahhed, G. The effect of weather conditions on the seasonal variation of physical activity. Aspetar Sports Med. J. 2015, 4, 228-231.

39. Tucker, P.; Gilliland, J. The effect of season and weather on physical activity: A systematic review. Public Health 2007, 121, 909-922. [CrossRef]

40. Edwards, N.M.; Myer, G.D.; Kalkwarf, H.J.; Woo, J.; Khoury, P.R.; Hewett, T.E.; Daniels, S.R. Outdoor Temperature, Precipitation, and Wind Speed Affect Physical Activity Levels in Children: A Longitudinal Cohort Study. J. Phys. Act. Health 2015, 12, 1074-1081. [CrossRef]

41. Quante, M.; Wang, R.; Weng, J.; Kaplan, E.R.; Rueschman, M.; Taveras, E.M.; Rifas-Shiman, S.L.; Gillman, M.W.; Redline, S. Seasonal and weather variation of sleep and physical activity in 12-14-year-old children. Behav. Sleep Med. 2019, 17, 398-410. [CrossRef] 